\title{
菌根共生网络嵌套性判定的零模型选择
}

\author{
林力涛 马克明*
}

中国科学院生态环境研究中心城市与区域生态国家重点实验室, 北京 100085 ; 中国科学院大学, 北京 100049

摘 要 零模型是判定网络嵌套性的重要依据, 菌根共生关系网络经常出现高度非对称性, 该文通过探究矩阵非对称变化对 基于不同零模型构建方法的网络嵌套性的影响, 试图为非对称网络零模型的选择提供依据。结果表明: 不同零模型保守性不 同, 增加限定条件减少零模型构建过程中的自由空间, 高度限定条件易导致第II类错误。高度非对称网络会增加基于完全随 机 $(\mathrm{r} 00)$ 零模型的矩阵温度 $(N T)$ 偏离、降低配对重叠度 $(N O D F)$ 偏离, 标准化指数z-score值显示网络非对称增加后有助于 $N T$ 和 $N O D F$ 显著性判定。行或列限定对非对称网络嵌套性判定的影响存在差异, 列限定 $(\mathrm{c} 0)$ 的网络嵌套性判定对网络非对称性变 化的响应规律与 $\mathrm{r} 00$ 零模型的响应趋势基本一致, 具有更低的嵌套性偏离和标准差值。行限定( $\mathrm{r} 0$, 包括行列限定(backtrack)) 零模型 $N T$ 值和 $N T$ 偏移随矩阵非对称性的变化保持稳定, 较之 $\mathrm{c} 0$ 零模型在高度非对称网络中呈现更低的 $N O D F$ 偏离值。选用完 全随机和限定零模型相结合的方法, 有助于更加准确判断非对称网络是否具有嵌套结构。高度非对称网络嵌套性判定中对行 属性特征比较敏感, 不同非对称性网络间嵌套性水平相比较时选用 $\mathrm{r} 0$ 零模型要优于 $\mathrm{r} 00$ 和 $\mathrm{c} 0$ 零模型。

关键词 关系网络; 嵌套性; 零模型; 网络非对称性

林力涛, 马克明 (2019). 菌根共生网络嵌套性判定的零模型选择. 植物生态学报, 43, 611-623. DOI: 10.17521/cjpe.2019.0065

\section{Selection of null models in nestedness pattern detection of highly asymmetric mycorrhizal networks}

LIN Li-Tao and MA Ke-Ming*

State Key Laboratory of Urban and Regional Ecology, Research Center for Eco-Environmental Sciences, Chinese Academy of Sciences, Beijing 100085, China; and University of Chinese Academy of Sciences, Beijing 100049, China

\section{Abstract}

Aims Null model is an important basis for nesting judgment. Highly asymmetric structures often appear in plant symbolic fungal networks. This study aims to explore the influence of matrix asymmetric changes on network nesting judgment.

Methods The study was conducted based on various null model construction methods.

Important findings Constraints vary with changing null models, with reducing null space when additional qualifications were added during null model establishment. Highly constrained nulls are prone to causing type II errors. Highly asymmetric networks increase matrix temperature $(N T)$ deviation based on random (Equiprobableequiprobable, r00) null model while reducing overlap and decreasing fill $(N O D F)$ deviation. Values of $z$-score show that highly asymmetric networks contribute to the significant determination level of $N T$ and $N O D F$. The impacts on the judgment of nestedness of asymmetric networks differ between row and column fixed null models. The effects of network asymmetry change on nesting detection based on column constrained (c0) nulls are similar to that of random null model, but with smaller nesting deviation and standard deviations. No significant differences in both $N T$ and $N T$ deviations were observed among different asymmetry networks based on the row fixed (r0) nulls, with a lower NODF deviation in highly asymmetric network based on c0 nulls. To more accurately determine whether the asymmetric networks would have nested structures, we recommend using a combination of random and constrained null models. Our results also demonstrate that the $\mathrm{r} 0$ null model performs better than either the r00 null model or the $\mathrm{c} 0$ null model when comparing nesting level of different asymmetric networks.

Key words interaction network; nestedness; null model; web asymmetry

收稿日期Received: 2019-03-25 接受日期Accepted: 2019-06-05

基金项目: 国家自然科学基金(31470481)。Supported by the National Natural Science Foundation of China (31470481)

* 通信作者Corresponding author (mkm@rcees.ac.cn) 
Lin LT, Ma KM (2019). Selection of null models in nestedness pattern detection of highly asymmetric mycorrhizal networks. Chinese Journal of Plant Ecology, 43, 611-623. DOI: 10.17521/cjpe.2019.0065

群落中物种之间通过相互作用(如捕食、竞争、 寄生、互惠等)彼此关联构成网络(Bascompte, 2010; Thebault \& Fontaine, 2010)。将网络分析方法应用于 生态学研究由来已久(陈水华和王玉军, 2004; Ulrich et al., 2009; 李中才等, 2011), 其中嵌套性是互惠共 生关系网络(传粉、种子传播、菌根共生等)的典型 特征(Ulrich et al., 2009; 方强和黄双全, 2012), 即相 互作用关系中相对特化种(specialist)相互作用的物 种是相对泛化种 (generalist)相互作用的物种子集的 作用模式。为对相互作用关系类型进行甄别, 需要 通过建立零模型(null model)来对网络嵌套结构进行 显著性检验(Bascompte et al., 2003; Ulrich \& Gotelli, 2007)。当前, 不同零模型构建方法均在传粉网络和 种子传播网络(具有较低的非对称性)嵌套性判定中 应用较为成熟，而基于不同零模型的网络非对称性 变化对嵌套性判定影响的研究相对较少。近年来, 高通量技术的进步使得微生物信息获取量呈指数式 增长(Liu et al., 2012; Poisot et al., 2013), 在构建菌 根共生关系网络时, 宿主植物物种数量与共生真菌 操作分类单元(OTUs)数量之间可能出现数量级的 差异(Bahram et al., 2014), 导致网络呈现高度的非 对称性。相较于传统近方形的二分网络, 高度非对 称网络对零模型构建自由空间具有较强的限定作用 (Chagnon et al., 2016)。因此, 明确矩阵非对称变化 对基于不同零模型嵌套性判定的影响规律有助于高 度非对称网嵌套性结构判定中选取适宜零模型, 提 高网络嵌套结构判定的精准度。

不同的零模型检验反映的生态学意义存在区别 (Atmar \& Patterson, 1993; Wright et al., 1997; Ulrich et al., 2009; Kennedy et al., 2014), 选取适当的零模 型有助于准确揭示类群间相互作用关系的维持机 制。目前, 基于被动取样的嵌套性判定的零模型选 择已经进行了较多的研究。如完全随机零模型认为 同一类群内的各元素以相同的概率与另一类群中任 意元素建立共生关系(Atmar \& Patterson, 1993); 在 传粉网络研究中, 数量多且(或)分布广泛的物种更 容易被调查观测到, 需要通过增加限定零模型构建 条件(行列限定)抵消由于被动取样对共生关系嵌套 性判定的影响(Bascompte et al., 2003); 在探究海岛 距离对鸟类栖息地嵌套性的影响时, 将海岛承载能
力作为零模型构建限定因子(Vázquez et al., 2009; 罗芳等, 2013; 张雪梅等, 2016)等。然而, 关于非对 称矩阵嵌套性判定中零模型的选择缺乏深入探究 (Blüthgen et al., 2007), Almeida-Neto等(2008)指出矩 阵形状、大小、填充度等因素均是影响矩阵嵌套性 度量值的重要因素, 矩阵非对称变化后对不同限定 零模型的构建自由度的影响程度存在差异(Chagnon et al., 2016)。因此, 选取适宜的零模型对于非对称 菌根共生关系网络嵌套性判定具有重要价值。当前, 在非对称网络属性特征方面已经进行了一些基础研 究, 如Blüthgen等(2007)对51个动植物间互惠网络 分析显示随矩阵非对称性增加网络特异性水平升高, Almeida-Neto等(2008)指出零模型构建方法是影响 不同形状随机矩阵嵌套性度量值保守性的重要因素, 但仍缺乏基于多种零模型选择的网络非对称变化对 嵌套性判定影响的研究。

目前, 已有相当多的研究显示宿主植物可与大 量不同真菌建立共生关系(Bahram et al., 2011; Gao et al., 2015), 即基于高通量测序结果的植物共生真 菌关系网络呈现高度非对称性 (Bahram et al., 2014)。同时，进行关系网络构建时也会面临相互作 用物种数量不保守情形, 如Toju等(2014)在构建外 生菌根(EM)、从枝菌根(AM)等关系子网络时面临共 生真菌物种数存在差异的问题。共生关系网络中, 物种连接点数是直接反映物种间相互作用关系强度 的重要指标, 然而针对不同形状、大小网络, 该指标 可能存在上限不保守的问题; 灰度填充度是表征相 互作用紧密程度的重要指标, 能够反映出当前网络 中相互作用关系距离潜在最大相互作用强度的程 度。此外, 我们对Atmar和Patterson (1995)研究中287 个网络分析显示, 行数相近的各组网络其灰度填充 度与非对称性之间相关性未达到显著水平(附录 I), 即行数相近时网络内相对作用强度随矩阵非对称性 变化差异不显著。为此, 本文以灰度填充度保守为 前提进行非对称网络的阐述, 假定行元素表征宿主 植物物种, 列元素表征共生真菌, 通过物种总数保 守和宿主植物物种总数保守两种方式构建不同非对 称性网络, 探究矩阵非对称变化对网络嵌套性判定 的影响及其在不同限定零模型中的表现, 为高度非对 称共生关系网络嵌套性判定零模型选择提供借鉴。 


\section{1 研究方法}

\section{1 关系矩阵的构建}

本研究中所有运算均在统计分析软件R 3.5.2 (https://cran.r-project.org)中实现。随机矩阵由vegan 2.5-4包(Oksanen et al., 2019)中nullmodel命令创 建, 用 method 参数选用完全随机 (equiprobable row-equiprobable column, r00)、行限定 (fixed row-equiprobable column, r0)、列限定(equiprobable row-fixed column, c0) 和行列限定(fixed row-fixed column, backtrack)等算法构建定性矩阵(附录II)。嵌 套性指数用bipartite 2.11包(Dormann et al., 2018)中 nested命令实现, method参数确定嵌套性指标类型。 本文中, 所有选用和构建的矩阵均假定行元素表征 植物、列元素表征菌根真菌, 通过改变列数/行数比 值来模拟关系网络中共生真菌OTUs分类单元数远 高于宿主植物物种数量的情形。

以填充度 $50 \%$ 、行列数 $10 \times 10$ 完全嵌套和均匀 分布的矩阵为初始矩阵(IM), 初步探析不同零模型 构建方法对矩阵嵌套性和反嵌套性判定的影响(附 录II)。在矩阵非对称变化对嵌套性判定影响中, 为 保证矩阵非对称变化后具有相近的嵌套性水平, 使 其更具可对比性, 本文选用了物种总数保守和宿主 植物物种总数保守两种网络构建方式:

(1) 物种总数保守: 以物种总数 60 , 行列数 $30 / 30 、 25 / 35 、 20 / 40 、 15 / 35 、 10 / 50 、 5 / 55$, 填充度 $50 \%$ 左右的 600 个(每水平 100 个)完全随机r00矩阵作 为初始矩阵，用以表征不同非对称性同时具有相同 嵌套水平的网络。

(2)宿主植物物种总数保守: 本研究选取 bipartite包中 5 个最接近方形互惠关系网络作为初始矩 阵(附录III), 假定行元素表征植物、列元素表征菌根
真菌, 基于初始矩阵通过列合并方式产生 1 倍、5倍、 10 倍的初始矩阵, 探究矩阵非对称性变化对网络嵌 套性判定的影响，及零模型选择对其的影响。通过列 合并的方式产生矩阵，虽然物种连接强度发生变化， 但其具有相同水平的相对连接强度，即网络具有相 同的灰度填充度水平。列合并非对称网络中共生真 菌和宿主植物在选择合作对象时与近方形网络具有 相同的作用格局，因而，用其来表征具有相同嵌套 性水平但不同非对称性水平的关系矩阵。

\section{2 嵌套性指标选择}

矩阵温度 $(N T)$ 和配对重叠度 $(N O D F)$ 是两种较 为常用的计算网络嵌套性的方法(表1)。 $T$ 由Atmar 和Patterson (1993) 提出, 是针对矩阵与相同大小且 填充度完全嵌套矩阵之间偏离的计算指标, 由于 $T$ 值随嵌套性增加而下降, 我们用 $N T=100-T$ 转换 来表征基于矩阵温度的嵌套性指标(Bascompte et al., 2003; Almeida-Neto et al., 2008)。调整矩阵行列 顺序使灰度单元格尽可能地排在矩阵的左上角, 辨 识矩阵中与其相应嵌套矩阵不同的“缺失”和“多余”, 计算与其相应最大程度嵌套矩阵的偏离:

$$
u_{i j}=\left(d_{i j} / D_{i j}\right)^{2}
$$

式中, $u_{i j}$ 为矩阵中位于 $i$ 行 $j$ 列的意外值(“缺失”和“多 余”); $D_{i j}$ 为贯穿 $i j$ 点对角线距离; $d_{i j}$ 为 $i j$ 点沿这条线的 特定距离。

$$
U=\frac{1}{f P} \sum_{i} \sum_{j} u_{i j}
$$

式中, $f$ 为行数, $P$ 为列数, $U$ 为总意外值, 为两次标准 化后的指标, 不受矩阵大小和性状约束; 矩阵完全 嵌套时 $U$ 值等于 0 , 矩阵完全无序化 $U_{\mathrm{m}} \approx 0.04145$ 。

$$
T=k U
$$

\begin{tabular}{|c|c|c|c|c|c|}
\hline $\begin{array}{l}\text { 指标 } \\
\text { Index }\end{array}$ & $\begin{array}{l}\text { 反映特征 } \\
\text { Underlying feature }\end{array}$ & $\begin{array}{l}\text { 下限保守 } \\
\text { Bottom boundary } \\
\text { conservative }\end{array}$ & $\begin{array}{l}\text { 上限保守 } \\
\text { Top boundary } \\
\text { conservative }\end{array}$ & $\begin{array}{l}\text { 零模型保守 } \\
\text { Null model } \\
\text { conservative }\end{array}$ & $\begin{array}{l}\text { 存在上限 } \\
\text { Top limit }\end{array}$ \\
\hline $\begin{array}{l}\text { 矩阵温度 } \\
\text { matrix temperature }(N T)\end{array}$ & $\begin{array}{l}\text { 矩阵中异常点数量、位置 } \\
\text { Relative abundance and position of unexpected } \\
\text { absences or presences in a matrix }\end{array}$ & 是 Yes & 是 Yes & 否 No & 是 Yes \\
\hline $\begin{array}{l}\text { 配对重叠度 } \\
\text { nestedness metric based on over- } \\
\text { lap and decreasing fill }(N O D F)\end{array}$ & $\begin{array}{l}\text { 矩阵配对重叠中非子集行列的数量及位置 } \\
\text { Percentage and position of columns (rows) overlaps } \\
\text { with other columns (rows) }\end{array}$ & 是 Yes & 是 Yes & 是 Yes & 是 Yes \\
\hline $\begin{array}{l}\text { 嵌套性偏移 } \\
\text { Nestedness deviation }\end{array}$ & $\begin{array}{l}\text { 网络与零模型的距离 } \\
\text { Distance deviates from null models }\end{array}$ & 是 Yes & 否 No & 是 Yes & 是 Yes \\
\hline $\begin{array}{l}\text { 标准化指数 } \\
\text { Standardized effect size }\end{array}$ & $\begin{array}{l}\text { 网络达到显著水平的难易程度 Significance of a } \\
\text { network deviates from null models }\end{array}$ & 是 Yes & 否 No & 是 Yes & 否 No \\
\hline
\end{tabular}

表1 嵌套性度量指标特征

Table 1 Characteristics of various nestedness metrics 
嵌套)到 $100^{\circ}$ (矩阵完全无序), 且不受矩阵大小约束。

配对重叠度由Almeida-Neto等(2008)基于矩阵 两个基本性质, 填充逐渐下降 $(D F)$ 和配对重叠 $(P O)$, 针对矩阵中物种相互配对重叠比例提出的计算指 标。调整矩阵物种填充(1's)尽可能地排在矩阵的左 上角, 矩阵中行总和、列总和逐渐递减; 假设调整 后的 $\mathrm{m} \times \mathrm{n}$ 矩阵, $i$ 行在 $j$ 行上方, $k$ 列在 $l$ 列左方, $M T$ 为 行或列的填充之和; 对任意两行, 如果 $M T_{j}<M T_{i}$, 则 $D F_{i j}$ 等于 100 , 否则 $D F_{i j}$ 为 0 ; 同样, 矩阵中任意两 列 $M T_{l}<M T_{k}$, 则 $D F_{k l}$ 等于 100 , 否则 $D F_{k l}$ 为 0 。列配对 重叠 $P O_{k l}$ 表示 $l$ 列中 1 's在 $k$ 列中具有相同 1 's所占的比 例, 行配对重叠 $P O_{i j}$ 表示 $j$ 行中1's在 $i$ 行中具有相同1's

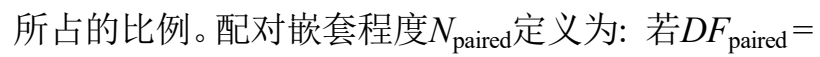
0 , 则 $N_{\text {paired }}=0$; 若 $D F_{\text {paired }}=100$, 则 $N_{\text {paired }}=P O$ 。

$$
N O D F=\frac{\sum N_{\text {paired }}}{\left[\frac{n(n-1)}{2}\right]+\left[\frac{m(m-1)}{2}\right]}
$$

式中, $N O D F$ 值的变化范围是从 0 (矩阵完全无序)到 100 (矩阵完全嵌套), 且不受矩阵大小约束。

为方便不同矩阵嵌套性对比, Ulrich和Gotelli (2007) 提出标准化指数 $(S E S)$ 记为 $z$-score $=(x-\mu) / \sigma$,
其中 $x$ 为实际观测值, $\mu$ 为 100 个模拟值矩阵嵌套性平 均值, $\sigma$ 为 100 个模拟矩阵嵌套性标准差。当 $z$-score 高于或低于 \pm 1.96 时达到统计学双尾检验 0.05 显著 水平, 认为该网络具有显著(反)嵌套结构。除直接的 显著水平比较之外, 这里将嵌套性偏移定义为矩阵 真实值与模拟矩阵平均值的差值, 能够量化显示 矩阵嵌套性值与零模型值数值差距(MontesinosNavarro et al., 2012)。

\section{2 结果}

\section{1 不同零模型嵌套性度量值变化特征}

不同零模型相比较, 高嵌套性和均匀分布(高 模块性)矩阵均表明行列频度(关系矩阵中非 0 单元 格的个数)限定零模型(backtrack)能够较大程度上保 留初始矩阵特征属性; 同时, 高度规整的矩阵具有 较小的零模型构建自由空间, 其标准差接近于 0 (图 $1)$ 。完全随机零模型( $(\mathrm{r} 00)$ 在嵌套性或非嵌套性判定 中更易达到显著水平，其嵌套性水平仅与初始矩阵 内部元素分布格局相关，以高嵌套或均匀分布矩阵 为初始矩阵构建 $\mathrm{r} 00$ 零模型具有相同的 $N T$ 值及 $N O D F$ 值。行 $(\mathrm{r} 0)$ 或列 $(\mathrm{c} 0)$ 频度限定零模型较 $\mathrm{r} 00$ 保守
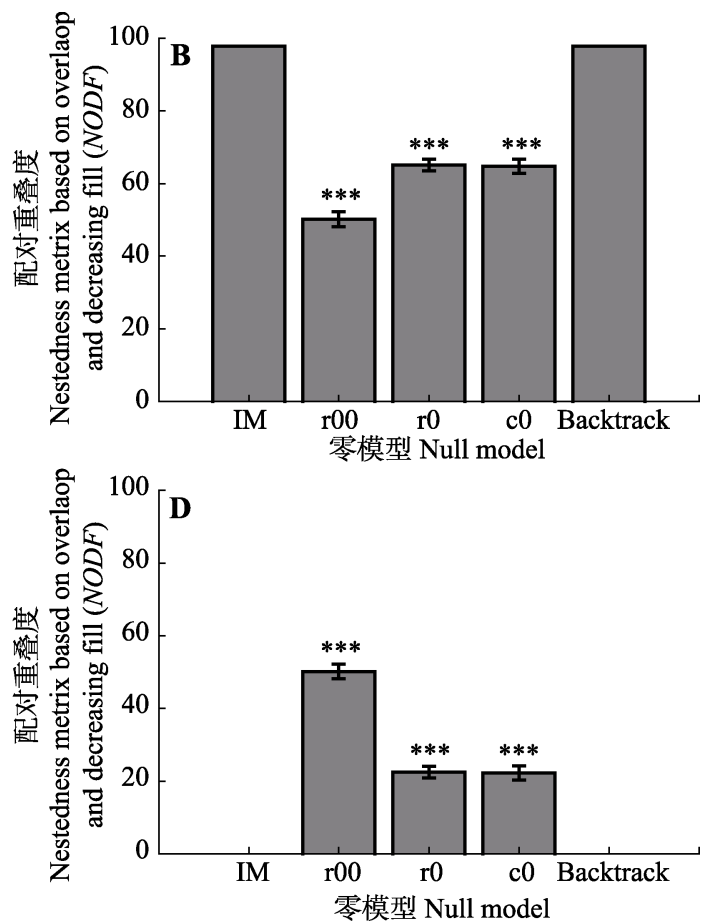

图 1 基于完全嵌套 $(\mathbf{A}, \mathbf{B})$ 和均匀分布 $(\mathbf{C}, \mathbf{D})$ 矩阵构建的不同零模型嵌套性特征(平均值土标准偏差)。Backtrack, 行列限定; c0, 列限定; IM, 初始矩阵; r0, 行限定; r00, 完全随机。***, 0.001 水平显著。

Fig. 1 Absolute values of nestedness from null models constructed based on fully nested $(\mathbf{A}, \mathbf{B})$ and uniformly distributed matrices $(\mathbf{C}, \mathbf{D})$ (mean $\pm S D$ ). Backtrack, fixed row-fixed column; c0, equiprobable row-fixed column; IM, initial matrix; r0, fixed rowequiprobable column; r00, equiprobable row-equiprobable column. ***, significant at 0.001 level.

www.plant-ecology.com 
性高, 表明矩阵需要更高的嵌套性(反嵌套性)特征 时与零模型对比才能达到显著水平, 如图1B中行 (列)频率总和限制零模型显著高于(低于)完全随机 零模型。
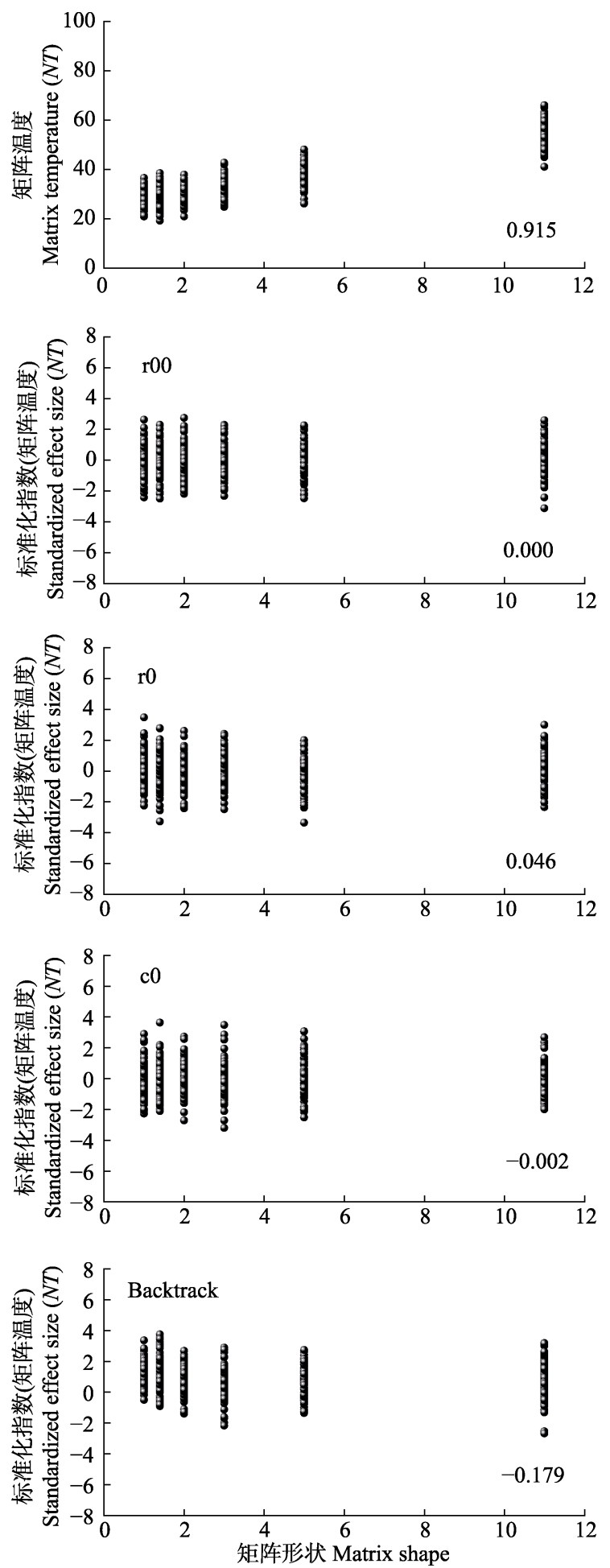

\section{2 矩阵行列属性对完全随机矩阵嵌套值的影响}

根据嵌套性的定义, 认为不同非对称性各组随 机矩阵具有相同的嵌套性显著水平。由图2可知, 不 同嵌套性度量指标对矩阵非对称性变化相应存在区
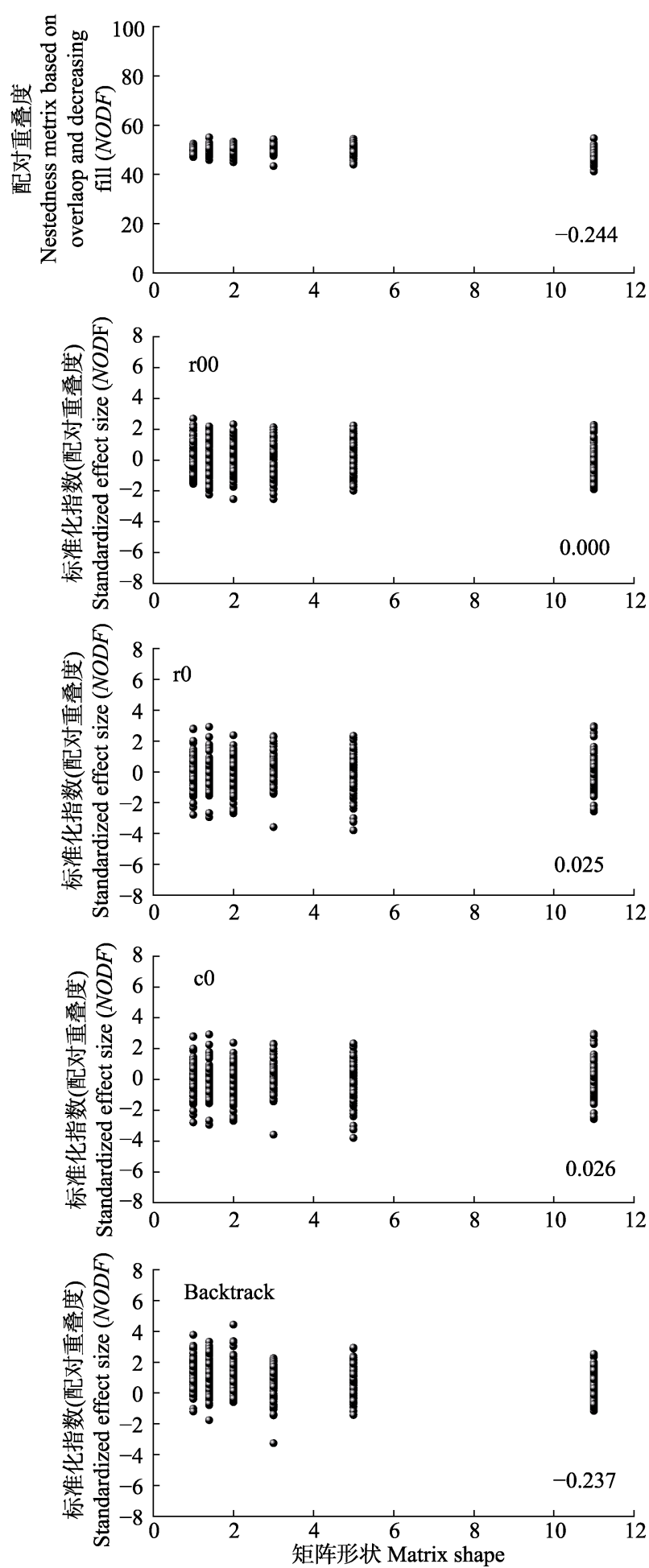

图2 矩阵形状变动对矩阵嵌套性指数影响。矩阵形状, 矩阵列数/矩阵行数。 Backtrack, 行列限定; $\mathrm{c} 0$, 列限定; $\mathrm{r} 0$, 行限定; $\mathrm{r} 00$, 完全随机。

Fig. 2 Effects of changes in matrix shape on nestedness metrics. Matrix shape, number of columns/number of rows. Backtrack, fixed row-fixed column; c0, equiprobable row-fixed column; r0, fixed row-equiprobable column; r00, equiprobable row-equiprobable column. 
别, $N T$ 随矩阵非对称性增大呈显著增加的趋势, 对 矩阵形状具有显著的依赖性; 而矩阵非对称性变化 对 $N O D F$ 指标无显著影响, 二者相关性未达到显著 水平。在嵌套显著水平判定中, 基于 $\mathrm{r} 00 、 \mathrm{r} 0 、 \mathrm{c} 0$ 零 模型的标准化两种度量指标 $z$-score指数随矩阵非对 称变化均能保持稳定, 然而基于高度限定的 bactrack零模型的 $z$-score指数随矩阵非对称性增加呈下
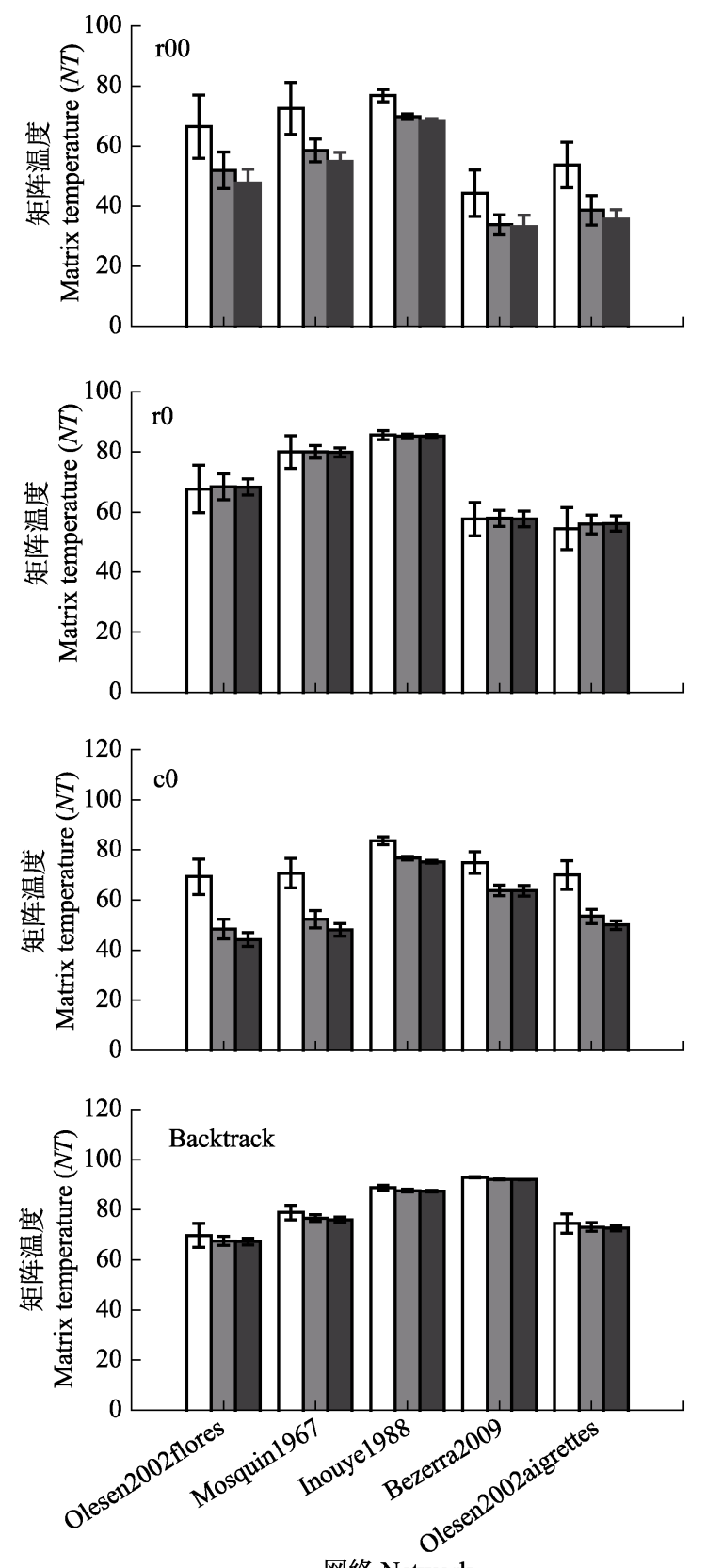

网络 Network
降的趋势。

\section{3 非对称性对不同零模型矩阵温度的影响}

行元素总数限定的网络中, 基于不同零模型构 建方法 $N T$ 值对矩阵非对称性变化的响应规律存在 差异(图3)。r0和backtrack零模型 $N T$ 值对矩阵非对称 变化响应不敏感; 而 $\mathrm{r} 00$ 和 $\mathrm{c} 0$ 零模型 $N T$ 值随矩阵非 对称性增加而降低, 为 $I M>5$ 倍 $>10$ 倍, 这可能是
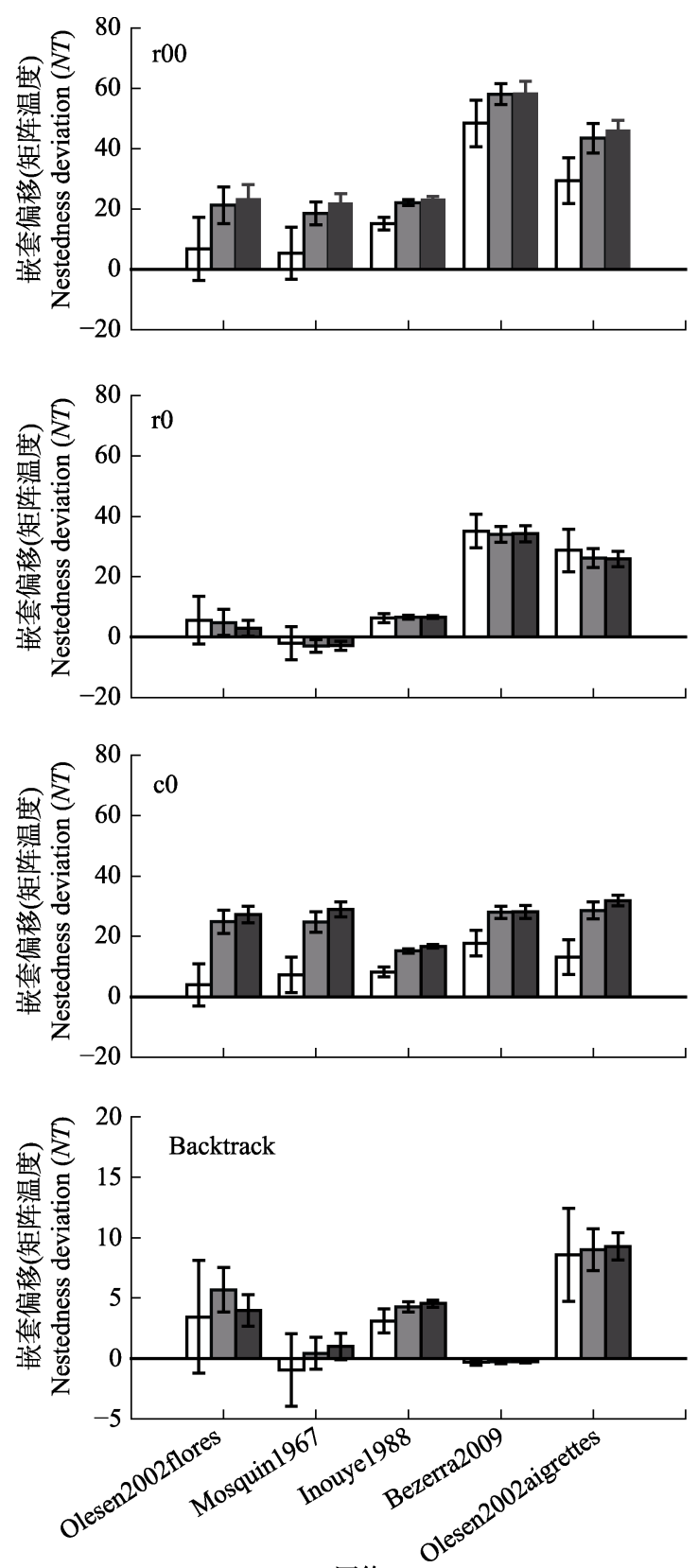

网络 Network

图3 非对称性变化对构建二分零模型矩阵温度 $(N T)$ 和 $N T$ 偏移的影响(平均值土标准偏差)。Backtrack, 行列限定; $\mathrm{c} 0$, 列限定; r0, 行限定; r00, 完全随机。IM, 初始矩阵; 5 times, 5倍列合并网络; 10 times, 10倍列合并网络。

Fig. 3 Effects of matrix asymmetric variations on matrix temperature $(N T)$ and $N T$ deviation based on different binary matrix construction methods (mean $\pm S D$ ). Backtrack, fixed row-fixed column; c0, equiprobable row-fixed column; r0, fixed row-equiprobable column; r00, equiprobable row-equiprobable column. IM, Initial matrix; 5 times, 5 times column merge network; 10 times, 10 times column merge network.

www.plant-ecology.com 
与矩阵属性特征改变相关(图1)。嵌套性偏移显示, r0 和backtrack构建矩阵的 $N T$ 偏移在非对称性发生变 化后保持稳定; 而 $\mathrm{r} 00$ 和 $\mathrm{c} 0$ 构建矩阵的 $N T$ 偏移值随 矩阵非对称性增加而逐渐增加, 与 $N T$ 值下降趋势相 对应。此外, $\mathrm{c} 0$ 零模型对不同对称性矩阵 $N T$ 偏移影 响存在差异, 与完全随机 $\mathrm{r} 00$ 方法相比, IM矩阵 $N T$ 降低 $(34.37 \pm 17.40) \%$, 而5 倍和10倍矩阵 $N T$ 偏移较 之 $\mathrm{r} 00$ 矩阵仅降低 $(13.40 \pm 16.34) \%$ 和 $(13.15 \pm$ $15.55) \%$ 。

标准化指数 $(z$-score) 显示, 不同零模型构建方 法对矩阵嵌套性显著性判定具有较大的影响(图 4)。如基于 $\mathrm{r} 00$ 零模型显著的 olesen2002flores 和 mosquin 1967 网络在对比 $\mathrm{r} 0$ 零模型时其 $z$-score接近 0 , 即行属性特征是导致网络矩阵嵌套性的因素, 对行 属性限定后其嵌套性未达到显著水平。参照 backtrack零模型的bezerra2009网络在与其他零模型 相比较时其嵌套显著水平发生转变, 较之 $\mathrm{r} 00$ 较高 的 $z$-score 指数以及 $\mathrm{r} 0 、 \mathrm{c} 0$ 达到显著嵌套水平, backtrack具有更高的矩阵嵌套判定保守性。随初始 矩阵非对称性增加, 基于 $\mathrm{r} 00$ 和 $\mathrm{c} 0$ 零模型 $z$-score呈增 加趋势; 而基于 $\mathrm{r} 0$ 零模型, 嵌套性中性的网络在非 对称性发生变化后 $z$-score指数基本稳定, 显著嵌套 的网络在非对称变化后 $z$-score指数呈增加趋势; 基 于backtrack构建方法 $z$-score指数变化与 $\mathrm{r} 0$ 具有相近 的变化趋势, 但其 $z$-score绝对值低于r0。

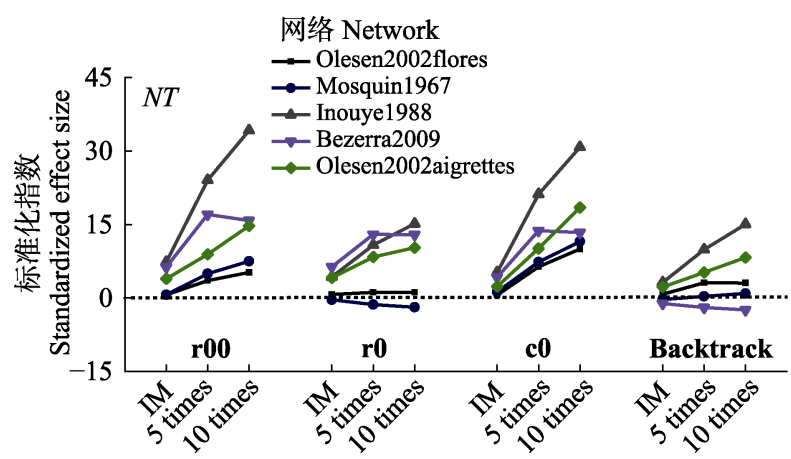

图 4 非对称性变化对矩阵温度标准化指数的影响。 Backtrack, 行列限定; c0, 列限定; r0, 行限定; r00, 完全随 机。IM, 初始矩阵; 5 times, 5倍列合并网络; 10 times, 10倍 列合并网络。

Fig. 4 Effects of matrix asymmetric variations on the standardized effect size ( $z$-score) of matrix temperature $(N T)$. Backtrack, fixed row-fixed column; c0, equiprobable row-fixed column; r0, fixed row-equiprobable column; r00, equiprobable row-equiprobable column. IM, initial matrix; 5 times, 5 times column merge network; 10 times, 10 times column merge network.

\section{4 非对称性对不同零模型配对重叠度的影响}

$N O D F$ 是基于配对重叠的嵌套性度量指标，不 同零模型构建方法 $N O D F$ 值对矩阵非对称性变化响 应亦存在差异(图5)。矩阵非对称性对 $\mathrm{r} 00$ 零模型 $N O D F$ 值无显著影响，而 $\mathrm{r} 0 、 \mathrm{c} 0$ 和backtrack零模型 $N O D F$ 值随矩阵非对称变化表现不同: 网络在 $\mathrm{r} 0$ 零 模型随对称性增大 $N O D F$ 值表现为增加趋势; 在 $\mathrm{c} 0$ 零模型中表现为下降趋势; backtrack零模型 $N O D F$ 值变化特征介于二者之间, 如 $\mathrm{r} 0$ 增幅较大的 olesen2002flores网络在backtrack零模型中随非对称 增加呈增大的趋势, $\mathrm{c} 0$ 降幅较大的 bezerra2009和 olesen2002aigrettes网络表现为下降趋势。结果显示, 基于 $\mathrm{c} 0$ 零模型的 $N O D F$ 偏移随网络非对称性变化基 本保持稳定，而基于r00、r 0 和backtrack 零模型的 $N O D F$ 偏移随网络非对称性变化呈下降的趋势。相 较于 $\mathrm{r} 0$ 构建方法 $N T$ 偏移基本稳定, NODF偏移随网 络非对称性增加呈现较大幅度的下降。基于 backtrack零模型的 $N O D F$ 偏移随网络非对称增加逐 渐降低至负值, 即呈现反嵌套模式。

标准化指数 $(z$-score) 显示, 不同零模型构建方 法的矩阵嵌套性显著水平可能发生改变, 如 inouye 1988 网络 $z$-score指数在 $\mathrm{r} 00 、 \mathrm{r} 0$ 和 $\mathrm{c} 0$ 零模型比 对中达到嵌套性显著嵌套水平, 而在backtrack零模 型比对中呈现随机或显著反嵌套结构。如图6所示, 随矩阵非对称性增加基于 $\mathrm{r} 00$ 和 $\mathrm{c} 0$ 零模型 $z$-score 呈 增加趋势, 而基于 $\mathrm{r} 0$ 方法网络矩阵在非对称性发生 变化后 $z$-score基本保持稳定。与 $N T$ 值 $z$-score不同, 基于 backtrack构建方法的 $N O D F$ 值 $z$-score 在矩阵 非对称性增加后对矩阵嵌套性判定中更为保守, 而对反嵌套性判定更为敏感, 如 backtrack方法 olesen2002aigrettes网络嵌套性的显著性发生转变, $N O D F$ 值 $z$-score低于 $N T$ 值 $z$-score。

\section{3 讨论}

\section{1 不同零模型在嵌套性判定中保守性的差异}

零模型是进行嵌套性判定的重要依据(Ulrich \& Gotelli, 2007; Ulrich et al., 2009), 通过零模型比较 可以看出: $r 00$ 零模型具有较高的构建自由空间, 受 初始矩阵内单元格嵌套性或反嵌套性分布格局影响 较小(图2), 仅与矩阵自身填充度相关(Almeida-Neto et al., 2008)。在探究菌丝网络中共生真菌在网络中作 用差别时, 通过与r00对比显示部分共生真菌在整个 

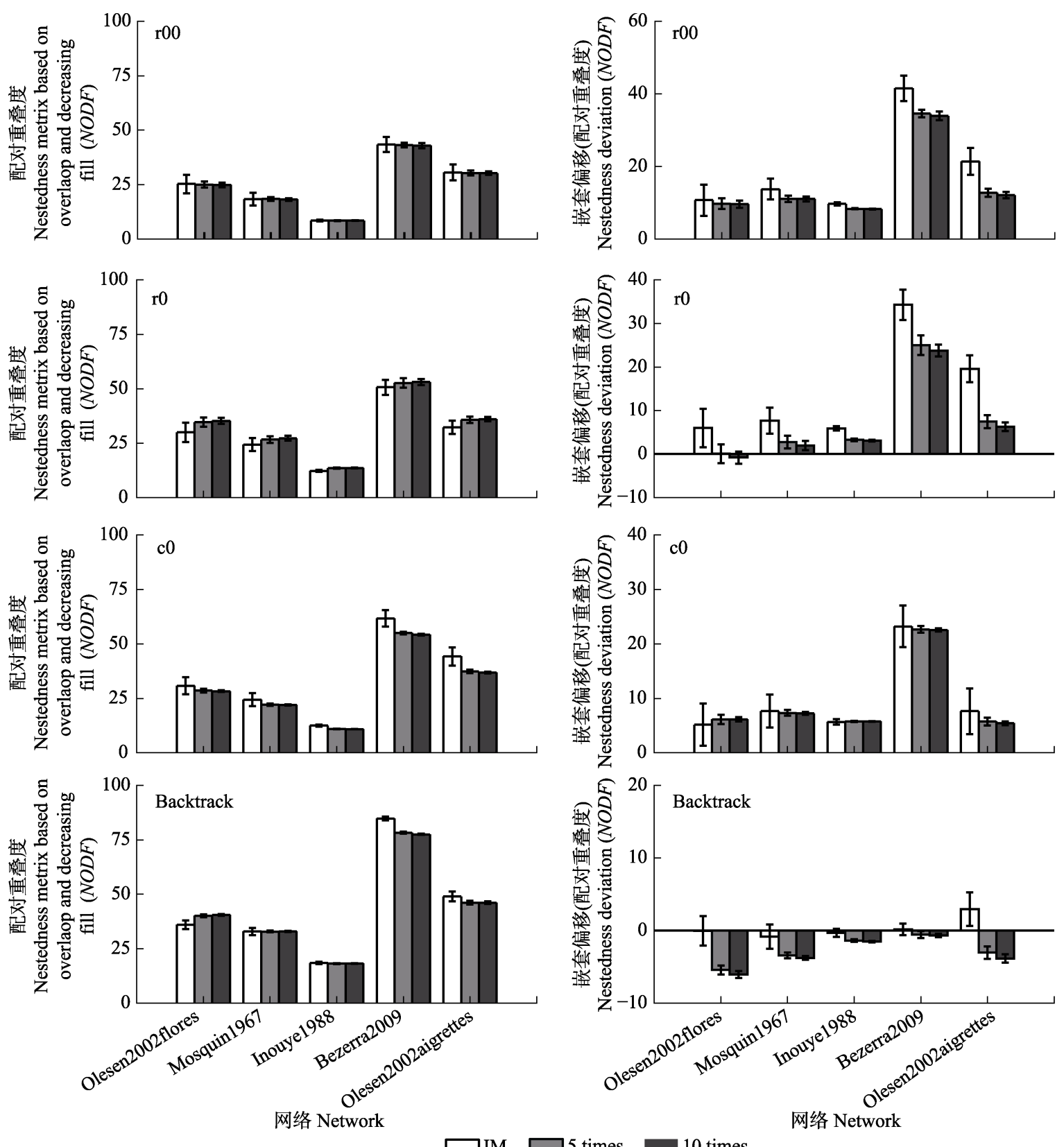

图5 非对称性变化对构建二分零模型配对重叠度 $(N O D F)$ 和 $N O D F$ 偏移影响(平均值土标准偏差)。Backtrack, 行列限定; $\mathrm{c} 0$, 列限定; r0, 行限定; r00, 完全随机。IM，初始矩阵; 5 times, 5倍列合并网络; 10 times, 10倍列合并网络。

Fig. 5 Effects of matrix asymmetric variations on nestedness metric based on overlap and decreasing fill (NODF) and $N O D F$ deviation based on different binary matrix construction methods (mean $\pm S D$ ). Backtrack, fixed row-fixed column; c0, equiprobable row-fixed column; r0, fixed row-equiprobable column; r00, equiprobable row-equiprobable column. IM, Initial matrix; 5 times, 5 times column merge network; 10 times, 10 times column merge network.

菌丝网络中可能起关键节点作用(Southworth et al., 2005)。通常认为嵌套性检验中最好的零假设可能是 所有的物种具有相同的出现概率 (Cook \& Quinn, 1998), 然而, 受取样因素的影响, 稀有物种之间的 相互作用往往难以被直接观测到(Vázquez et al., 2009)。因此, 需要通过增加零模型行(列)限定条件来 抵消被动取样对嵌套性判定的影响(Gotelli, 2000)。近 方形的传粉网络或种子传播网络中, 植物限定的 $\mathrm{r} 0$
和传粉昆虫或食果动物限定的 $\mathrm{c} 0$ 零模型较完全随机 $\mathrm{r} 00$ 零模型保守性高, 应用该方法生成的随机矩阵能 够保留初始矩阵中植物(传粉昆虫或食果动物)的部 分特征, 其嵌套性度量值较之 $\mathrm{r} 00$ 零模型更为接近初 始矩阵真实值(图1，图3，图5)。限定零模型应用较为 广泛, 鸟类岛屿生物地理学研究中常利用该种方法 将海岛承载能力作为构建零模型的限定因素用于探 究距离因子对鸟类栖息地嵌套性的影响(Vázquez www.plant-ecology.com 


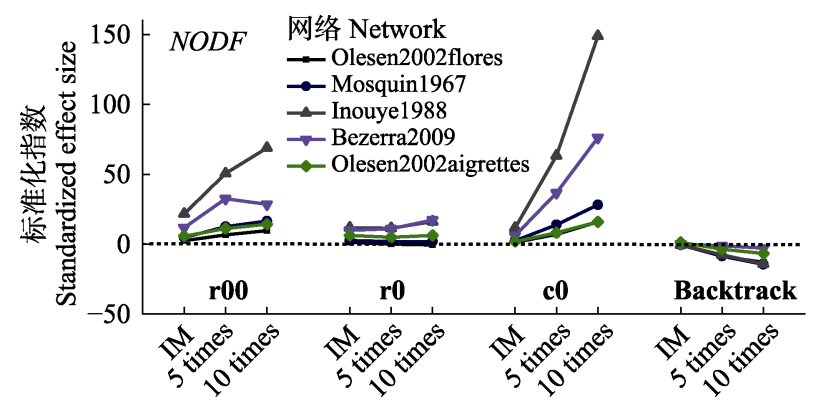

图6 非对称性变化对矩阵配对重叠度标准化指数的影响。 Backtrack, 行列限定; c0, 列限定; r0, 行限定; r00, 完全随 机。IM, 初始矩阵; 5 times, 5 倍列合并网络; 10 times, 10 倍 列合并网络。

Fig. 6 Effects of matrix asymmetric variations o $z$-scores of on the standardized effect size ( $z$-score) of nestedness metric based on overlap and decreasing fill $(N O D F)$. Backtrack, fixed row-fixed column; c0, equiprobable row-fixed column; r0, fixed row-equiprobable column; r00, equiprobable rowequiprobable column. IM, Initial matrix; 5 times, 5 times column merge network; 10 times, 10 times column merge network.

et al., 2009; 罗芳等, 2013; 张雪梅等, 2016)。此外, 当二分网络相互作用两大类群其中一个类群的各物 种为均等取样时, 即该类群在构建共生关系网络时 不受种群数量差异的影响, 可使用 $\mathrm{r} 0$ 或 $\mathrm{c} 0$ 零模型去 除被动取样因素影响并进行网络显著性的检验 (Ulrich \& Gotelli, 2013)。

高度限定的backtrack零模型较之 $\mathrm{r} 0$ 和 $\mathrm{c} 0$ 方法又 具有更高的保守性, 适用于相互作用两大类群在构 建关系网络时均受被动取样影响的情况, 如传粉网 络调查取样过程中数量多且(或)分布广泛的植物或 传粉昆虫种更容易被调查观测到(Bascompte et al., 2003), 但该方法对于高度规整网络嵌套性检验中具 有一定局限性(Chagnon et al., 2016), 易引发第II类 错误(图1), 即显著嵌套的关系网络在统计分析过程 中判定为未达到嵌套性显著水平, 过低估计网络嵌 套结构的显著性水平。嵌套性是互惠关系网络的典 型特征, 基于不同零模型构建方法网络的嵌套性水 平可能会发生改变, 如Bascompte等(2003)对 52 个食 果、传粉网络嵌套性分析发现当参照零模型由r00变 为backtrack时有 6 个网络嵌套性水平发生改变; Kennedy等(2014)的研究也表明backtrack零模型在嵌套 性判断中具有较高的保守性, 基于backtrack方法未 达到显著的网络在与 $\mathrm{r} 0$ 零模型对比后达到嵌套性显 著水平。本研究亦显示出类似规律, 如bezerra2009网 络嵌套性 $N T$ 在 $\mathrm{r} 00 、 \mathrm{r} 0 、 \mathrm{c} 0$ 构建方法对比中达到显著 水平, 而与backtrack零模型对比后未达到显著水平;
甚者, $N O D F$ 嵌套性衡量方法中 bezerra2009网络在 backtrack零模型对比后呈现显著的反嵌套水平。

\section{2 非对称网络与传统近方形网络在嵌套性判定 中的不同}

矩阵嵌套性度量指标对矩阵属性变化的响应存 在差异(Ulrich \& Gotelli, 2007), 完全零模型大小及 形状改变后 $N O D F$ 值无显著变化, 而 $N T$ 值与矩阵形 状呈显著正相关关系(图2), 与Almeida-Neto等(2008) 的研究结果相一致。宿主植物总数保持不变时, r00 零模型 $N T$ 值随矩阵非对称性增加呈降低的趋势, 而 $N O D F$ 值基本保持稳定(图3, 图5)。两种嵌套性方法 相比较, 随矩阵有序程度降低至完全随机分布, $N T$ 值在测定高度有序水平矩阵时发挥出色, 但其在完 全随机分布时 $N T$ 度量值不保守; 而 $N O D F$ 值在低有 序水平度量时发挥出色, 矩阵随机分布时具有相同 的 $N O D F$ 度量值(图7, 图8)。虽然 $N T$ 和 $N O D F$ 对矩阵 非对称性变化相应存在差异, 但在与 $\mathrm{r} 00$ 零模型进行 显著性检验时, 网络非对称变化对各组矩阵 $N T$ 和 $N O D F$ 显著性检验均无显著的影响(图2), 二者的 $z$-score指数均能呈现相等的下限, 但随矩阵有序程 度增加, $z$-score指数上限不保守, 近方形网络 $z$-score 指数要高于非对称性网络(图9; 表1), 即基于 $\mathrm{r} 00$ 的 $z$-score指数可在显著性判定中进行应用, 在不同非 对称性的高度有序网络间进行比较时仍具有一定局 限性。

增加限定条件的零模型构建方法能够去除取样 环节中被动取样因素的影响, 有助于更加保守地判 定物种间相互作用的本质(Ulrich et al., 2009)。同时, 增加限定条件的同时将会极大减少零模型构建过程 中的自由空间(Chagnon et al., 2016), 对于低填充度、 高非对称网络选用限定零模型不能有效地随机化共 生关系矩阵。物种总数一定的随机网络中, 基于 backtrack零模型 $N T$ 和 $N O D F$ 值的 $z$-score指数在提高 判定保守性的同时, 容易引发第II类错误; 从backtrack零模型构建自由度来看, $N T$ 和 $N O D F$ 值的 $z$-score 指数与矩阵非对称性负相关, 表明高度非对称网络 较之近方形网络保留更多原始矩阵的信息(图2)。本 研究显示, 利用backtrack 零模型对高度规整网络进 行显著性验证时亦存在较大的局限性(图1, 图5), 完 全随机的零模型是较好的替代选择(Ulrich \& Gotelli, 2013), 利用 $\mathrm{r} 00$ 方法生成矩阵 $N O D F$ 值随矩阵非对称 性变化基本稳定, $N T$ 值随矩阵非对称性增大而减小, 

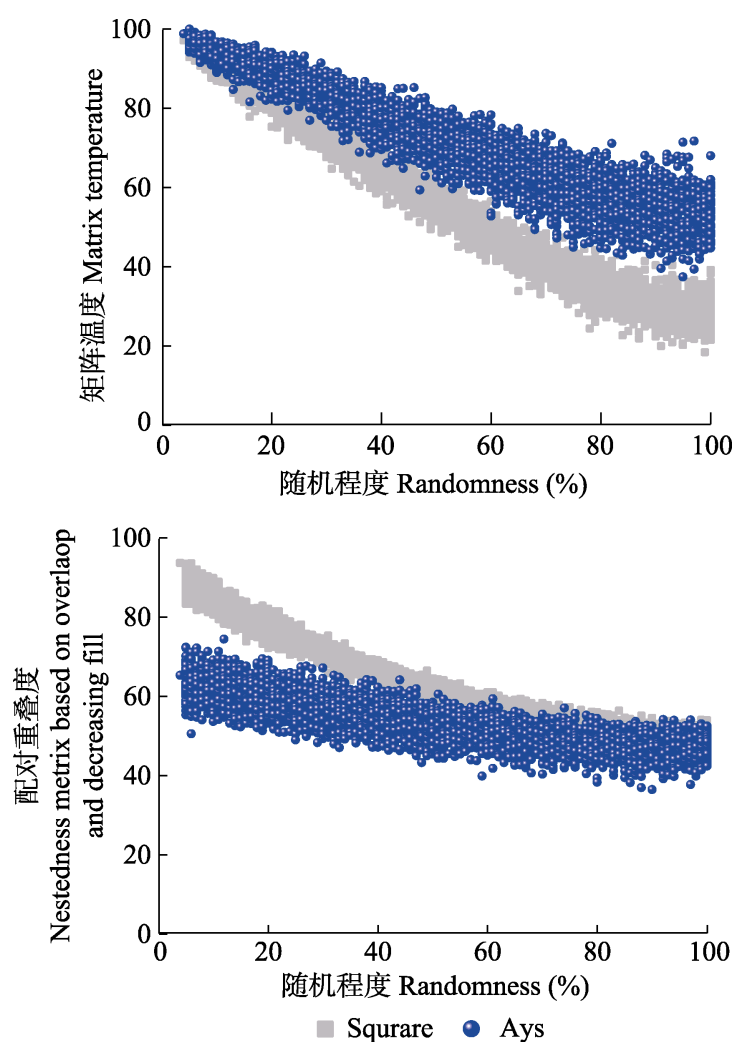

图7 矩阵随机化程度对嵌套性度量指标的影响。Square, 对 称网络, 行列数 $30 / 30 ;$ Asy, 非对称网络, 行列数 $5 / 55$ 。随机 化程度, 网络中无序单元格的数量/单元格总数量。随机程度 包含 4:100 (\%)共计97水平, 每水平 50 个重复, 共计4 850矩 阵。

Fig. 7 Effects of the level of matrix randomness on nestedness metrics. Square, symmetric networks with rows and columns 30/30; Asy, asymmetric networks with rows and columns 5/55. Randomness, percentage of disordered cells deviates from fully nested matrix. Data contains a total of 4850 matrices, with 4:100 (\%) randomness degree 97 levels of, 50 repetitions per level.

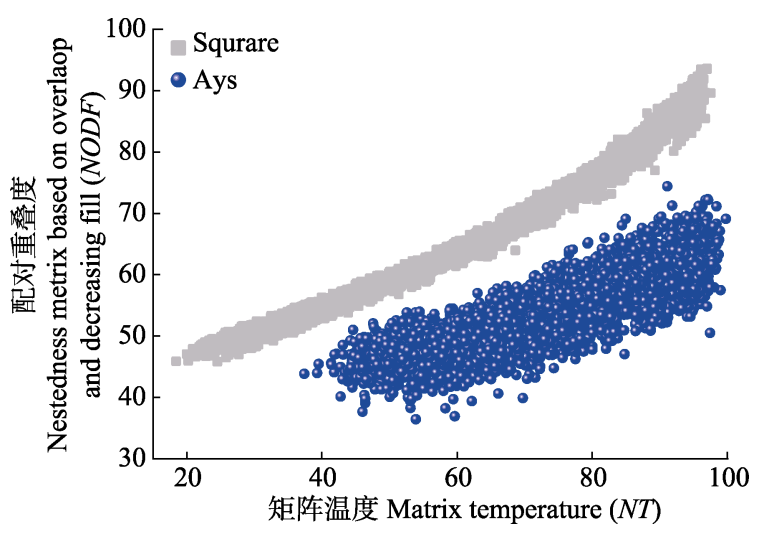

图 8 矩阵温度 $(N T)$ 与配对重叠度 $(N O D F)$ 间的相关性。 Square, 对称网络, 行列数 $30 / 30$; Asy, 非对称网络, 行列数 $5 / 55$ 。随机化程度, 网络中无序单元格的数量/单元格总数 量。随机程度包含 4:100 (\%)共计97水平, 每水平50个重复, 共计4 850矩阵。

Fig. 8 Correlation between matrix temperature $(N T)$ and nestedness metric based on overlap and decreasing fill (NODF). Square, symmetric networks with rows and columns 30/30; Asy, asymmetric networks with rows and columns 5/55. Randomness, percentage of disordered cells deviates from fully nested matrix. Data contains a total of 4850 matrices, with 4:100 (\%) randomness degree 97 levels of, 50 repetitions per level.

$N T$ 偏移和 $z$-score 随非对称性增大而增大, 均未对网 络嵌套性判定产生负效应。

行(列)限定对矩阵非对称变化后嵌套性判定影 响不同, 本研究中, 行限定的 $\mathrm{r} 0$ 和backtrack零模型 $N T$ 值和 $N T$ 偏移随网络非对称性增加无显著变化, 而列 限定 $\mathrm{c} 0$ 零模型 $N T$ 值和 $N T$ 偏移变化与 $\mathrm{r} 00$ 零模型结果 相近(图3)。基于矩阵温度的嵌套性判定方法在高度 非对称矩阵嵌套性判定中对行属性特征更敏感, 即
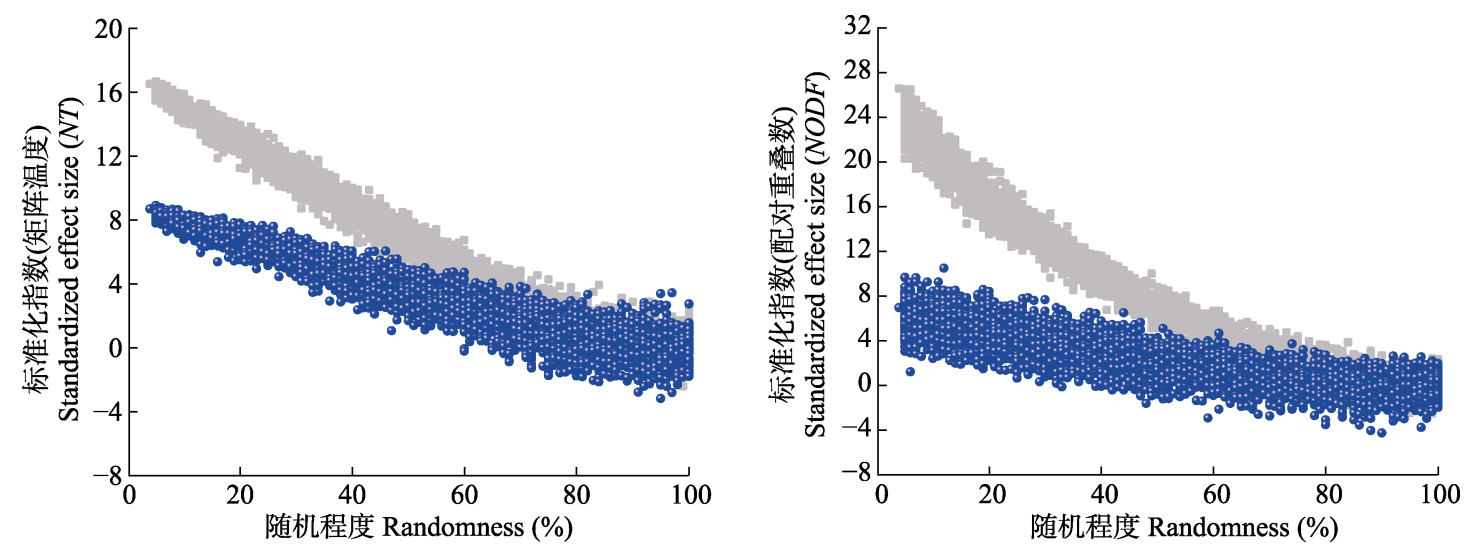

- Squrare $\bullet$ Ays

图9 矩阵随机化程度对嵌套标准化指标影响。Square, 对称网络, 行列数 $30 / 30 ;$ Asy, 非对称网络, 行列数 $5 / 55$ 。随机化程度, 网络中无序单元格的数量/单元格总数量。随机程度包含 $4: 100(\%)$ 共计 97 水平, 每水平 50 个重复, 共计 4850 矩阵。NT, 矩阵温 度; $N O D F$, 配对重叠度。

Fig. 9 Effects of the level of matrix randomness on the standardized effect size of nestedness. Square, symmetric networks with rows and columns 30/30; Asy, asymmetric networks with rows and columns 5/55. Randomness, percentage of disordered cells deviates from fully nested matrix. Data contains a total of 4850 matrices, with 4:100 (\%) random-ness degree 97 levels of, 50 repetitions per level. $N T$, matrix temperature; $N O D F$, nestedness metric based on overlap and decreasing fill. 
宿主植物行属性特征是影响非对称网络零模型构建 自由度的主导因素。 $N O D F$ 值对矩阵大小及形状不敏 感, 但基于 $\mathrm{r} 00$ 方法生成矩阵 $N O D F$ 偏移随矩阵非对 称性增加呈下降趋势, 与 $N T$ 指标趋势相反。这主要 是由于二者对待调整后矩阵中互为子集列(行)处理 方式不同(表1), NT强调异常点出现点数量和位置, 而 $N O D F$ 将相同列(行)结构视为妨碍矩阵嵌套的结构 (Ulrich et al., 2009)。随非对称性增加基于backtrack 零模型的 $N O D F$ 偏移表现出与 $\mathrm{r} 00$ 方法相反的结果, 如基于backtrack方法的bezerra2009网络嵌套偏移显 示由嵌套向反嵌套方向发生转变(图5)。此外, 矩阵非 对称性变化对基于不同零模型的 $z$-score指数影响存 在差异, 行限定的 $\mathrm{r} 0$ 方法网络矩阵非对称性变化 $z$-score基本稳定, 列限定 $z$-score指数随矩阵非对称增 加而呈现升高趋势。这可能与不同零模型生成矩阵 的标准差存在差异相关联, $\mathrm{c} 0$ 零模型构建自由度低于 $\mathrm{r} 00$ 零模型导致 $\mathrm{c} 0$ 零模型生成随机矩阵的标准差相对 较小, 当标准差的变化超过嵌套性偏移的变化时 $z$-score指数发生相应改变。

\section{3 单网络嵌套性的判定及多网络间的比较}

菌根非对称网络嵌套性判定中, 兼顾保守型的 同时也需要警惕第II类错误的发生, 可以采用多个零 模型相结合的方法进行验证。同时, 集合种群嵌套性 研究指出, 对于可能影响物种分布的因素(如生境大 小、隔离程度及其质量等)都是当嵌套性在统计上被 证明以后才考虑的问题(刘灿然等, 2002)。完全随机 零模型(r00), 假定同一类群内所有物种具有相等概 率与另一类群内任一物种建立联系, 具有较高的零 模型构建自由度(图2), 可以先将菌根非对称网络通 过与 $\mathrm{r} 00$ 零模型比较判定网络的嵌套性情况, 然后对 嵌套性的成因进行解析。经由 $\mathrm{r} 00$ 检验后达到嵌套性 显著的网络仍无法确定相互作用的双方是否存在彼 此选择的倾向性(Ulrich et al., 2009), 被动取样亦是 导致网络嵌套性的可能因素。后续嵌套性检验中, 可 通过构建限定零模型来去除物种的出现频率差异影 响(Bahram et al., 2011), 探究导致网络出现嵌套性结 构的原因。在检验过程中, 行或列限定零模型对高度 非对称网络嵌套性判定的影响存在差异(图3, 图5), 这主要与矩阵本身宿主植物行或共生真菌列自由度 空间差异有关。高度非对称矩阵嵌套性判定中对宿 主植物行属性特征更敏感(Beiler et al., 2015), 与r00 方法相比呈现更低的嵌套性偏离值(图3, 图5)。不同
于昆虫传粉网络、动物种子传播网络, 在植物-共生 真菌网络研究中由宿主植物频度差异部分产生的影 响能够在取样过程中予以控制(Ulrich \& Gotelli, 2013), 因此, 被动取样影响主要来自于共生真菌出 现频率的差异, 可选用 $\mathrm{c} 0$ 零模型对网络中共生真菌 物种出现频率进行限定, $\mathrm{c} 0$ 零模型较大程度地保留了 r00方法的特征, 具有较高的零模型构建自由度(图 3)。如果不同零模型嵌套性判定结果存在差异且限定 零模型方差值很小时, 可以谨慎选用 $\mathrm{r} 00$ 零模型结果 进行解释, 同时辅以物种累计曲线来阐述被动取样 在网络构建中的影响。当物种累计曲线达到饱和时, 则认为取样量能够涵盖该研究区内所有的物种, 不 存在由于数量少而未被监测到的物种, 即被动取样 效应对共生关系网络结构无影响。

通常, $N T$ 和 $N O D F$ 值均能够直观地反映相互作 用关系网络的有序水平, 且 $N O D F$ 对于关系矩阵大 小、性状变化不敏感, 能够较好地指示不同网络的有 序水平差异。然而, 网络间相互作用关系对网络结构 的贡献很难判断比较, 相关指示指标也相对较少。在 与零模型比较中容易确定网络是否具有显著嵌套结 构, 判定过程中嵌套性偏移和 $z$-score指数均能定量 化反映当前网络结构与随机情况的差异。本研究中 5 组网络结果均显示, 基于行限定零模型的 $N T$ 偏移值 随网络非对称性变化保持稳定(图3), 而NT标准化的 $z$-score指数由于随机矩阵方差差异随非对称性增加 呈现增大的趋势(附录I; 图4)。同时, 基于行限定零 模型 $N O D F$ 标准化 $z$-score指数亦显示随矩阵非对称 性变化维持不变(图5)。因此, 非对称性差异较大的网 络间进行嵌套性水平比较时, 鉴于非对称性网络中 行属性因素较强的限定作用, 选择 $\mathrm{r} 0$ 零模型要优于 $\mathrm{r} 00$ 和 $\mathrm{c} 0$ 零模型。

\section{4 结论}

高度非对称关系网络嵌套性判定, 选择零模型 强调保守性同时要注意零模型构建的自由空间, r00 和 $\mathrm{c} 0$ 方法均是不错的选择。选用 $\mathrm{r} 00$ 和限定零模型相 结合的方法, 有助于更加准确地判断非对称关系网 络是否嵌套, 揭示类群水平物种间相互作用的本质。 行或列限定零模型对高度非对称网络嵌套性判定的 影响存在差异, 矩阵嵌套性判定中对行属性特征更 敏感, 不同非对称性网络间嵌套性水平相比较时选 用 $\mathrm{r} 0$ 零模型要优于 $\mathrm{r} 00$ 和 $\mathrm{c} 0$ 零模型。 


\section{参考文献}

Almeida-Neto M, Guimarães P, Guimarães Jr PR, Loyola RD, Ulrich W (2008). A consistent metric for nestedness analysis in ecological systems: Reconciling concept and measurement. Oikos, 117, 1227-1239.

Atmar W, Patterson BD (1993). The measure of order and disorder in the distribution of species in fragmented habitat. Oecologia, 96, 373-382.

Atmar W, Patterson BD (1995). The Nestedness Temperature Calculator: A Visual Basic Program, Including 294 Presence-absence Matrices. AICS Research, University Park, NM and Field Museum, Chicago.

Bahram M, Harend H, Tedersoo L (2014). Network perspectives of ectomycorrhizal associations. Fungal Ecology, 7, 70-77.

Bahram M, Põlme S, Kõljalg U, Tedersoo L (2011). A single European aspen (Populus tremula) tree individual may potentially harbour dozens of Cenococcum geophilum ITS genotypes and hundreds of species of ectomycorrhizal fungi. FEMS Microbiology Ecology, 75, 313-320.

Bascompte J (2010). Structure and dynamics of ecological networks. Science, 329, 765-766.

Bascompte J, Jordano P, Melian CJ, Olesen JM (2003). The nested assembly of plant-animal mutualistic networks. Proceedings of the National Academy of Sciences of the United States of America, 100, 9383-9387.

Beiler KJ, Simard SW, Durall DM (2015). Topology of tree-mycorrhizal fungus interaction networks in xeric and mesic Douglas-fir forests. Journal of Ecology, 103, 616-628.

Bezerra ELS, Machado IC, Mello MAR (2009). Pollination networks of oil-flowers: A tiny world within the smallest of all worlds. Journal of Animal Ecology, 78, 1096-1101.

Blüthgen N, Menzel F, Hovestadt T, Fiala B, Blüthgen N (2007). Specialization, constraints, and conflicting interests in mutualistic networks. Current Biology, 17, 341-346.

Chagnon PL, U'Ren JM, Miadlikowska J, Lutzoni F, Elizabeth Arnold A (2016). Interaction type influences ecological network structure more than local abiotic conditions: Evidence from endophytic and endolichenic fungi at a continental scale. Oecologia, 180, 181-191.

Chen SH, Wang YJ (2004). Nestedness pattern of insular community assemblages and its applications. Chinese Journal of Ecology, 23, 81-87. [陈水华, 王玉军 (2004). 岛屿群落组成的嵌套格局及其应用. 生态学杂志, 23 , 81-87.]

Cook RR, Quinn JF (1998). An evaluation of randomization models for nested species subsets analysis. Oecologia, 113, 584-592.

Dormann CF, Gruber B, Fruend J, Gruber B (2018). Bipartite:
Analysis of bipartite ecological webs. R package version 2.11. https://cran.r-project.org/package=bipartite.

Fang Q, Huang SQ (2012). Progress in pollination networks: Network structure and dynamics. Biodiversity Science, 20, 300-307. [方强, 黄双全 (2012). 传粉网络的研究进展: 网络的结构和动态. 生物多样性, 20, 300-307.]

Gao C, Zhang Y, Shi NN, Zheng Y, Chen L, Wubet T, Bruelheide H, Both S, Buscot F, Ding Q, Erfmeier A, Kühn P, Nadrowski K, Scholten T, Guo LD (2015). Community assembly of ectomycorrhizal fungi along a subtropical secondary forest succession. New Phytologist, 205, $771-785$.

Gotelli NJ (2000). Null model analysis of species co-occurrence patterns. Ecology, 81, 2606-2621.

Inouye DW, Pyke GH (1988). Pollination biology in the Snowy Mountains of Australia: Comparisons with montane Colorado, USA. Australian Journal of Ecology, 13, 191-205.

Kennedy P, Nguyen NH, Cohen H, Peay K (2014). Missing checkerboards? An absence of competitive signal in Alnusassociated ectomycorrhizal fungal communities. Peer $J, 2$, e686. DOI: $10.7717 /$ peerj.686.

Li ZC, Xu JY, Wu CY, Zhang Y (2011). A review of studies using ecological network analysis. Acta Ecologica Sinica, 31, 5396-5405. [李中才, 徐俊艳, 吴昌友, 张渏 (2011). 生态网络分析方法研究综述. 生态学报, 31, 5396-5405.]

Liu CR, Ma KP, Chen LZ (2002). Nestedness: Methods, mechanisms and implications for biological conservation. Acta Phytoecologica Sinica, 26, 68-72. [刘灿然, 马克平, 陈灵芝 (2002). 嵌套性: 研究方法、形成机制及其对生 物保护的意义. 植物生态学报, 26, 68-72.]

Liu KL, Porras-Alfaro A, Kuske CR, Eichorst SA, Xie G (2012). Accurate, rapid taxonomic classification of fungal large-subunit rRNA genes. Applied and Environmental Microbiology, 78, 1523-1533.

Luo F, Pan Y, Lu CH (2013). Structural characteristics of plant-animal mutualistic networks and related affecting factors. Chinese Journal of Ecology, 32, 2179-2185. [罗 芳, 潘扬, 鲁长虎 (2013). 动植物互惠网络结构及影响 因素. 生态学杂志, 32, 2179-2185.]

Montesinos-Navarro A, Segarra-Moragues JG, Valiente-Banuet A, Verdú M (2012). The network structure of plantarbuscular mycorrhizal fungi. New Phytologist, 194, 536-547.

Mosquin T, Martin JEH (1967). Observations on the pollination biology of plants on Melville Island, N.W.T, Canada. $\mathrm{Ca}$ nadian Field Naturalist, 81, 201-205.

Oksanen J, Blanchet FG, Friendly M, Kindt R, Legendre P, McGlinn D, Minchin PR, O'Hara RB, Simpson GL, Solymos P, Stevens MHH, Szoecs E, Wagner H (2019). Vegan: Community ecology package. R package version 2.5-4. https://CRAN.R-project.org/package=vegan. Cited:

www.plant-ecology.com 
2019-03-20

Olesen JM, Eskildsen LI, Venkatasamy S (2002). Invasion of pollination networks on oceanic islands: Importance of invader complexes and endemic super generalists. Diversity and Distributions, 8, 181-192.

Poisot T, Péquin B, Gravel D (2013). High-throughput sequencing: A roadmap toward community ecology. Ecology and Evolution, 3, 1125-1139.

Southworth D, He XH, Swenson W, Bledsoe CS, Horwath WR (2005). Application of network theory to potential mycorrhizal networks. Mycorrhiza, 15, 589-595.

Thebault E, Fontaine C (2010). Stability of ecological communities and the architecture of mutualistic and trophic networks. Science, 329, 853-856.

Toju H, Guimarães PR, Olesen JM, Thompson JN (2014). Assembly of complex plant-fungus networks. Nature Communications, 5, 5273. DOI: 10.1038/ncomms6273.

Ulrich W, Almeida-Neto M, Gotelli NJ (2009). A consumer's guide to nestedness analysis. Oikos, 118, 3-17.
Ulrich W, Gotelli NJ (2007). Null model analysis of species nestedness patterns. Ecology, 88, 1824-1831.

Ulrich W, Gotelli NJ (2013). Pattern detection in null model analysis. Oikos, 122, 2-18.

Vázquez DP, Blüthgen N, Cagnolo L, Chacoff NP (2009). Uniting pattern and process in plant-animal mutualistic networks: A review. Annals of Botany, 103, 1445-1457.

Wright DH, Patterson BD, Mikkelson GM, Cutler A, Atmar W (1997). A comparative analysis of nested subset patterns of species composition. Oecologia, 113, 1-20.

Zhang XM, Han XF, Liu LW, Xu AC (2016). Influencing factors of the nested distribution of butterfly assemblages in the Zhoushan Archipelago, China. Biodiversity Science, 24, 321-331. [张雪梅, 韩徐芳, 刘立伟, 徐爱春 (2016). 舟山群岛蝶类群落嵌套分布格局及其影响因素. 生物 多样性, 24, 321-331.]

责任编委: 周淑荣 责任编辑: 李 敏 实习编辑: 赵 航

附录I 行数相近矩阵形状变化对填充度的影响

Supplement I Effects of matrix shape variations on connectance http://www.plant-ecology.com/fileup/1005-264X/PDF/cjpe.2019.0065-S1.pdf

附录II 不同零模型构建方法及其特征

Supplement II Null model construction methods and characteristics http://www.plant-ecology.com/fileup/1005-264X/PDF/cjpe.2019.0065-S2.pdf

附录III 5个互惠共生关系网络及基本特征

Supplement III Five mutually-symbiotic relationship networks and their basic properties http://www.plant-ecology.com/fileup/1005-264X/PDF/cjpe.2019.0065-S3.pdf 
林力涛, 马克明 (2019). 菌根共生网络嵌套性判定的零模型选择. 植物生态学报, 43, 611-623. DOI: 10.17521/cjpe.2019.0065

Lin LT, Ma KM (2019). Selection of null models in nestedness pattern detection of highly asymmetric mycorrhizal networks. Chinese Journal of Plant Ecology, 43, 611-623. DOI: 10.17521/cjpe.2019.0065

$5 \quad$ http://www.plant-ecology.com/CN/10.17521/cjpe.2019.0065

6

附录I 行数相近矩阵形状变化对填充度的影响。

8

Supplement I Effects of matrix shape variations on connectance.

9
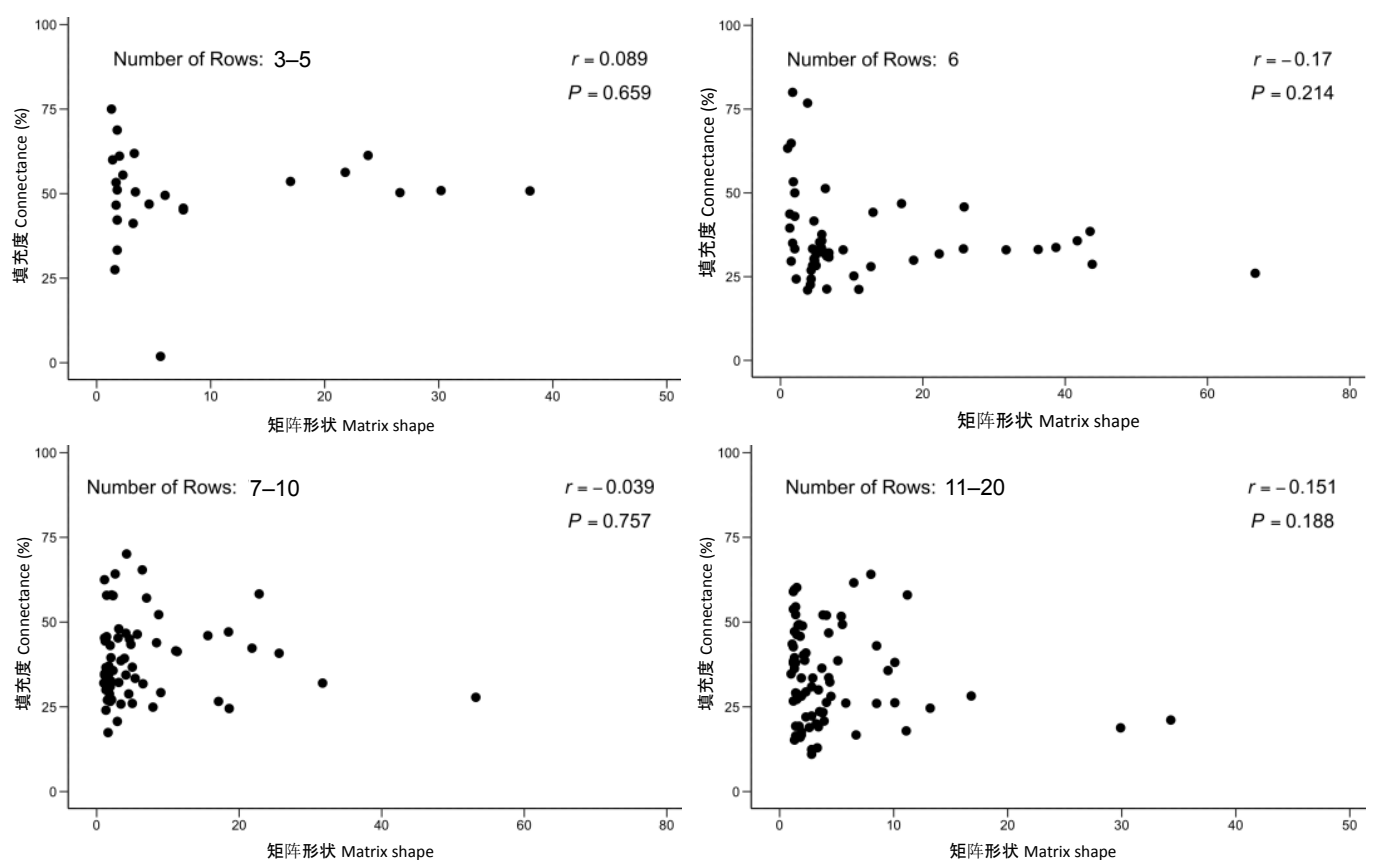

11

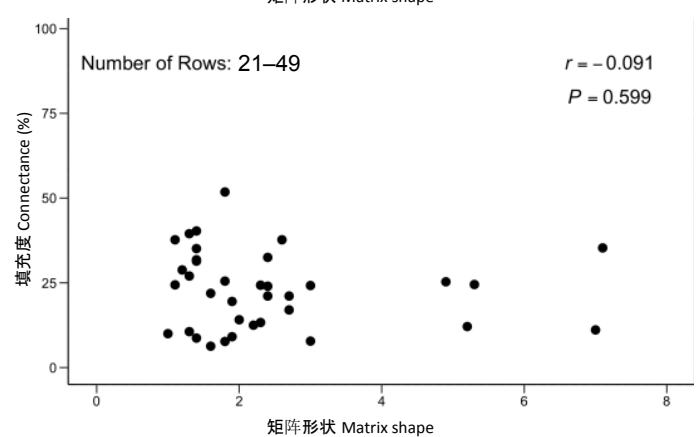

12

矩阵形状, 矩阵列数/矩阵行数。数据来源于 Atmar \& Patterson (1995)。

Matrix shape, number of columns / number of rows. Using 287 data sets provided by Atmar \& Patterson (1995).

参考文献

Atmar W, Patterson BD (1995). The Nestedness Temperature Calculator: A Visual Basic Program, Including 294 Presence-absence Matrices. AICS Research, University Park, NM and Field Museum, Chicago. 
林力涛, 马克明 (2019). 菌根共生网络嵌套性判定的零模型选择. 植物生态学报, 43, 611623. DOI: 10.17521/cjpe.2019.0065

Lin LT, Ma KM (2019). Selection of null models in nestedness pattern detection of highly asymmetric mycorrhizal networks. Chinese Journal of Plant Ecology, 43, 611-623. DOI: 10.17521/cjpe.2019.0065

http://www.plant-ecology.com/CN/10.17521/cjpe.2019.0065

附录II 不同零模型构建方法及其特征

Supplement II Null model construction methods and characteristics

\begin{tabular}{|c|c|c|c|c|}
\hline $\begin{array}{l}\text { 零模型 } \\
\text { Null model }\end{array}$ & $\begin{array}{l}\text { 限定条件 } \\
\text { Constraints }\end{array}$ & $\begin{array}{l}\text { 定量矩阵 } \\
\text { Quantitative } \\
\text { matrix }\end{array}$ & $\begin{array}{l}\text { 构建方法 } \\
\text { Method of construction }\end{array}$ & $\begin{array}{l}\text { 参考文献 } \\
\text { Reference }\end{array}$ \\
\hline r00 & 矩阵填充度 Connectance & 否 No & $\begin{array}{l}\text { 节点等概率 } \\
\text { Equiprobability among cells }\end{array}$ & Atmar \& Patterson (1993) \\
\hline r0 & $\begin{array}{l}\text { 矩阵填充度、行频度总和 } \\
\text { Connectance, row frequencies }\end{array}$ & 否 No & $\begin{array}{l}\text { 行等概率 } \\
\text { Equiprobability among rows }\end{array}$ & Patterson \& Atmar (1986) \\
\hline $\mathrm{c} 0$ & $\begin{array}{l}\text { 矩阵填充度、列频度总和 } \\
\text { Connectance, column frequencies }\end{array}$ & 否 No & $\begin{array}{l}\text { 列等概率 } \\
\text { Equiprobability among colums }\end{array}$ & Jonsson (2001) \\
\hline backtrack & $\begin{array}{l}\text { 矩阵填充度、行频度总和、列频 } \\
\text { 度总和 } \\
\text { Connectance, row frequencies, } \\
\text { column frequencies }\end{array}$ & 否 No & $\begin{array}{l}\text { 随机填充后矫正至行、列频度保 } \\
\text { 守 } \\
\text { Filling randomly, then } \\
\text { backtracking with constraints both } \\
\text { for row and column frequencies }\end{array}$ & Bascompte et al. (2003) \\
\hline
\end{tabular}

行(列)频度总和, 行(列)中灰度单元格(非0单元格)的数量保持不变。

Keep the number of gray cells (non-zero cells) in the row (column) being consistent.

\section{参考文献}

Atmar W, Patterson BD (1993). The measure of order and disorder in the distribution of species in fragmented habitat. Oecologia, 96, 373-382.

Bascompte J, Jordano P, Melian CJ, Olesen JM (2003). The nested assembly of plant-animal mutualistic networks. Proceedings of the National Academy of Sciences of United States of America, 100, 9383-9387.

Jonsson BG (2001). A null model for randomization tests of nestedness in species assemblages. Oecologia, 127, 309-313.

Patterson BD, Atmar W (1986). Nested subsets and the structure of insular mammalian faunas and archipelagoes. Biological Journal of the Linnean Society, 28, 65-82. 
林力涛, 马克明 (2019). 菌根共生网络嵌套性判定的零模型选择. 植物生态学报, 43, 611623. DOI: 10.17521/cjpe.2019.0065

Lin LT, Ma KM (2019). Selection of null models in nestedness pattern detection of highly asymmetric mycorrhizal networks. Chinese Journal of Plant Ecology, 43, 611-623. DOI: 10.17521/cjpe.2019.0065

http://www.plant-ecology.com/CN/10.17521/cjpe.2019.0065

附录III 5个互惠共生关系网络及基本特征

Supplement III Five mutually-symbiotic relationship networks and their basic properties

\begin{tabular}{lccccccc}
\hline $\begin{array}{l}\text { 网络 } \\
\text { Network }\end{array}$ & $\begin{array}{c}\text { 行数 } \\
\text { Rows }\end{array}$ & $\begin{array}{c}\text { 列数 } \\
\text { Columns }\end{array}$ & $\begin{array}{c}\text { 填充度 } \\
\text { Connectance }\end{array}$ & $\begin{array}{c}\text { 连接度 } \\
\text { Link per } \\
\text { specie }\end{array}$ & $\begin{array}{c}\text { 矩阵温度 } \\
N T\end{array}$ & $\begin{array}{c}\text { 配对 } \\
\text { 重叠度 }\end{array}$ & $\begin{array}{c}\text { 参考文献 } \\
\text { Reference }\end{array}$ \\
\hline olesen2002flores & 10 & 12 & 0.25 & 1.36 & 73.26 & 35.96 & Olesen et al. (2002) \\
$\begin{array}{l}\text { mosquin1967 } \\
\text { inouye1988 }\end{array}$ & 11 & 18 & 0.19 & 1.31 & 77.93 & 32.07 & Mosquin \& Martin (1967) \\
bezerra2009 & 41 & 83 & 0.08 & 2.16 & 91.96 & 84.93 & Inouye \& Pyke (1988) \\
olesen2002aigrettes & 13 & 13 & 0.42 & 2.73 & 92.71 & 76.88 & Bezerra et al. (2009) \\
\hline
\end{tabular}

$N T$, matrix temperature; $N O D F$, nestedness metric based on overlap and decreasing fill.

\section{参考文献}

Olesen JM, Eskildsen LI, Venkatasamy S (2002). Invasion of pollination networks on oceanic islands, importance of invader complexes and endemic super generalists. Diversity and Distributions, 8, 181-192.

Mosquin T, Martin JEH (1967). Observations on the pollination biology of plants on Melville Island, N.W.T, Canada. Canadian Field Naturalist, 81, 201-205.

Inouye DW, Pyke GH (1988). Pollination biology in the snowy mountains of Australia-Comparisons with Montane Colorado, USA. Australian Journal of Ecology, 13, 191-210.

Bezerra ELS, Machado IC, Mello MAR (2009). Pollination networks of oil-flowers: A tiny world within the smallest of all worlds. Journal of Animal Ecology, 78, 1096-1101. 